\title{
Theoretical Analysis of Neutron and X-ray Scattering Data on ${ }^{3} \mathrm{He}$
}

\author{
E. Krotscheck • M. Panholzer
}

Received: 2 September 2010 / Accepted: 23 November 2010 / Published online: 7 December 2010

(C) The Author(s) 2010. This article is published with open access at Springerlink.com

\begin{abstract}
X-ray scattering experiments on bulk liquid ${ }^{3} \mathrm{He}$ (Albergamo et al. in Phys. Rev. Lett. 99:205301, 2007; Schmets and Montfrooij in Phys. Rev. Lett. 100:239601, 2008; Albergamo et al. in Phys. Rev. Lett. 100:239602, 2008) have indicated the possibility of the existence of a sharp collective mode at large momentum transfers. We address this issue within a manifestly microscopic theory of excitations in a Fermi fluid that can be understood as proper generalization of the time-honored theory of Jackson, Feenberg, and Campbell (Jackson in Phys. Rev. A 8:1529, 1973; Feenberg in Theory of Quantum Fluids, 1969; Chang and Campbell in Phys. Rev. B 13:3779, 1976) of excitations in ${ }^{4} \mathrm{He}$. We show that both neutron and X-ray data can be well explained within a theory where the high momentum excitations lie in fact inside the particle-hole continuum. "Pair fluctuations" contribute a sharpening of the mode compared to the random phase approximation (RPA). When the theoretical results are convoluted with the experimental resolution, the agreement between theory and $\mathrm{X}$-ray data is quite good.
\end{abstract}

Keywords Theory of liquid ${ }^{3} \mathrm{He} \cdot$ Dynamic structure function $\cdot$ Neutron-scattering X-ray scattering

\section{Introduction}

The helium fluids ${ }^{3} \mathrm{He}$ and ${ }^{4} \mathrm{He}$ are the prime examples of strongly correlated quantum many-body systems. Governed by a simple Hamiltonian, yet very dense, they

E. Krotscheck $(\varangle) \cdot$ M. Panholzer

Institut für Theoretische Physik, Johannes Kepler Universität, 4040 Linz, Austria

e-mail: Eckhard.Krotscheck@jku.at

E. Krotscheck

Department of Physics, University at Buffalo SUNY, Buffalo, NY 14260, USA 
have been studied for decades and still offer surprises leading to new insights. It is fair to say that understanding the helium fluids lies at the core of understanding other strongly correlated systems.

Liquid ${ }^{3} \mathrm{He}$ is for many reasons the more challenging substance for both, theoretical and experimental investigations. Key information on the dynamics of the system is provided by the dynamic structure function $S(q ; \omega)$. Experimentally, the dynamic structure function of ${ }^{3} \mathrm{He}$ is mostly determined by neutron scattering. The results are well documented in a book [1], the theoretical and experimental understanding a decade ago has been summarized in [2]. Recent inelastic X-ray scattering experiments have led to a controversy on the evolution of the zero sound mode at intermediate wave-vectors [3-5]. It is one of the purposes of this paper to examine this issue on the basis of microscopic many body theory.

The ground state of liquid ${ }^{4} \mathrm{He}$ is well understood from both diagrammatic manybody techniques and simulation methods, see [6] for a collection of pedagogical articles. Building upon pioneering work by Jackson, Feenberg, and Campbell [7-9], recent developments [10-12] have brought manifestly microscopic theories of ${ }^{4} \mathrm{He}$ to a level where quantitative predictions for the excitation spectrum are possible up to momentum transfers well beyond the roton minimum.

We have recently generalized the theory of [7-9] to Fermions [13]. From the theoretical point of view, the major technical problem is to deal with the multitude of exchange effects in an efficient manner; we have formulated a tractable strategy for including pair-excitation effects in Fermi fluids. This paper is concerned with a theoretical study of excitations and damping in normal liquid ${ }^{3} \mathrm{He}$ at atomic wave lengths based on that theory.

\section{Dynamic Many-Body Theory}

\subsection{Equations of Motion}

This section gives a very brief compilation of the techniques developed in [13]. We deliberately refrain from any explanation and refer to the original work and earlier pedagogical material [6] for details.

Microscopic many-body theory starts from a phenomenological Hamiltonian for $N$ interacting fermions,

$$
H=-\sum_{i} \frac{\hbar^{2}}{2 m} \nabla_{i}^{2}+\sum_{i<j} v\left(\left|\mathbf{r}_{i}-\mathbf{r}_{j}\right|\right) .
$$

For strong interactions, correlated basis functions (CBF) theory [8] has proved to be an efficient and accurate method for obtaining ground state properties. It starts with a variational wave function of the form

$$
\left|\Psi_{\mathbf{o}}\right\rangle=\frac{F\left|\Phi_{\mathbf{o}}\right\rangle}{\left\langle\Phi_{\mathbf{o}}\left|F^{\dagger} F\right| \Phi_{\mathbf{0}}\right\rangle^{1 / 2}},
$$


where $\Phi_{\mathbf{0}}(1, \ldots, i, \ldots, N)$ is a model state, normally a Slater-determinant, and " $i$ " is short for both spatial and $v$ discrete (spin and/or isospin) degrees of freedom. The correlation operator $F(1, \ldots, N)$ is suitably chosen to describe the important features of the interacting system. Most practical and successful is the Jastrow-Feenberg [8] form

$$
\begin{aligned}
& F(1, \ldots, N) \\
& \quad=\exp \left\{\frac{1}{2}\left[\sum_{1 \leq i<j \leq N} u_{2}\left(\mathbf{r}_{i}, \mathbf{r}_{j}\right)+\sum_{1 \leq i<j<k \leq N} u_{3}\left(\mathbf{r}_{i}, \mathbf{r}_{j}, \mathbf{r}_{k}\right)+\ldots\right]\right\} .
\end{aligned}
$$

The $u_{n}\left(\mathbf{r}_{1}, \ldots, \mathbf{r}_{n}\right)$ are made unique by requiring them to vanish for $\left|\mathbf{r}_{i}-\mathbf{r}_{j}\right| \rightarrow \infty$ ("cluster property").

From the wave function (2.2), (2.3), the energy expectation value

$$
H_{\mathbf{o}, \mathbf{o}} \equiv\left\langle\Psi_{\mathbf{o}}|H| \Psi_{\mathbf{o}}\right\rangle
$$

can be calculated either by simulation or by integral equation methods. The hierarchy of Fermi-Hypernetted-Chain (FHNC) approximations is compatible with the optimization problem, i.e. with determining the optimal correlation functions $u_{n}\left(\mathbf{r}_{1}, \ldots, \mathbf{r}_{n}\right)$ through functionally minimizing the energy

$$
\frac{\delta H_{\mathbf{o}, \mathbf{o}}}{\delta u_{n}\left(\mathbf{r}_{1}, \ldots, \mathbf{r}_{n}\right)}=0 .
$$

The dynamics of the system is treated with the logical generalization of the wave function (2.2), (2.3) by writing the response of the system to a weak and time dependent external field $H_{\text {ext }}(t)=\sum_{i} \delta h_{\text {ext }}\left(\mathbf{r}_{i} ; t\right)$ in the form

$$
\begin{aligned}
|\Psi(t)\rangle & =\frac{1}{\sqrt{\mathcal{N}}} e^{-\mathrm{i} E_{0} t / \hbar}\left|\Psi_{0}(t)\right\rangle, \\
\left|\Psi_{0}(t)\right\rangle & =F e^{\frac{1}{2} \delta U(t)}\left|\Phi_{0}\right\rangle, \quad \mathcal{N} \equiv\left\langle\Psi_{0}(t) \mid \Psi_{0}(t)\right\rangle,
\end{aligned}
$$

where $F$ is taken from the ground state calculation, and $\delta U(t)$ is a sum of $n$-particle$n$-hole excitation operators:

$$
\delta U(t)=\sum_{p h} \delta u_{p h}^{(1)}(t) a_{p}^{\dagger} a_{h}+\frac{1}{2} \sum_{p p^{\prime} h h^{\prime}} \delta u_{p p^{\prime} h h^{\prime}}^{(2)}(t) a_{p}^{\dagger} a_{p^{\prime}}^{\dagger} a_{h^{\prime}} a_{h}+\cdots
$$

As a convention, we will label "particle" (unoccupied) and "hole" (occupied) states with $p, p^{\prime}$ and $h, h^{\prime}$, respectively. Discrete degrees of freedom are suppressed.

Equations of motion for the $\delta u^{(i)}(t)$ are derived from the linearized action principle $[14,15]$

$$
\delta \int d t \mathcal{L}(t)=\delta \int d t\left\langle\Psi(t)\left|H+H_{\text {ext }}(t)-\mathrm{i} \hbar \frac{\partial}{\partial t}\right| \Psi(t)\right\rangle=0 .
$$

The analytic and diagrammatic manipulations to bring the resulting equations of motion into a numerically tractable form are lengthy and delicate [13], but the result 
are surprisingly simple and can be cast into the familiar form of the time-dependent Hartree-Fock (TDHF) theory [16] with energy dependent effective interactions. The induced density fluctuations is expanded in terms of matrix elements of the density operator in the non-interacting system, $\rho_{0, p h}^{F}(\mathbf{r})=\langle h|\delta \hat{\rho}(\mathbf{r})| p\rangle$.

$$
\delta \rho(\mathbf{r} ; \omega)=\frac{1}{2} \sum_{p h}\left[\rho_{0, p h}^{\mathrm{F}}(\mathbf{r}) \delta c_{p h}^{(+)}(\omega)+\rho_{0, p h}^{\mathrm{F} *}(\mathbf{r}) \delta c_{p h}^{(-)}(\omega)\right]
$$

The amplitudes $\delta c_{p h}^{( \pm)}(\omega)$ are related by the THDF equations to the matrix elements $h_{i j}=\left\langle i\left|\delta h_{\text {ext }}\right| j\right\rangle$ of the external field.

$$
\begin{gathered}
\left(\begin{array}{cc}
\left(\hbar \omega-e_{p h}\right) \delta_{p, p^{\prime}} \delta_{h, h^{\prime}}+V_{p h, p^{\prime} h^{\prime}}^{(A)}(\omega) & V_{p p^{\prime} h h^{\prime}, 0}^{(B)}(\omega) \\
V_{0, p p^{\prime} h h^{\prime}}^{(B)}(\omega) & -\left(\hbar \omega+e_{p h}\right) \delta_{p, p^{\prime}} \delta_{h, h^{\prime}}+V_{p^{\prime} h^{\prime}, p h}^{(A)}(\omega)
\end{array}\right) \\
\times\left(\begin{array}{c}
\delta c_{p h}^{(+)} \\
\delta c_{h p}^{(-)}
\end{array}\right)=2\left(\begin{array}{c}
h_{p h} \\
h_{h p}
\end{array}\right),
\end{gathered}
$$

where $e_{p h}=t(p)-t(h)$ is the particle-hole excitation energy of the non-interacting system and $t(q)=\hbar^{2} q^{2} / 2 m$ is the kinetic energy.

Thus, the machinery of microscopic many-body theory has led to a definition of the effective interactions $V_{p h, p^{\prime} h^{\prime}}^{(A)}(\omega)$ and $V_{p p^{\prime}, h h^{\prime}}^{(B)}(\omega)$ in terms of the correlation operator $F$ and the underlying microscopic Hamiltonian.

\subsection{Local Interactions}

Much of our qualitative understanding of the dynamics of interacting quantum fluids is based on the "random phase approximation" (RPA). In that approximation, the density-density response function is of the form

$$
\chi(q ; \omega)=\frac{\chi_{0}(q ; \omega)}{1-\tilde{V}_{\mathrm{p}-h}(q) \chi_{0}(q ; \omega)}
$$

Here, $\chi_{0}(q ; \omega)$ is the Lindhard function, and $\tilde{V}_{\mathrm{p}-h}(q)$ an appropriately defined static "particle-hole interaction" or "pseudo-potential [17]". It is related to the dynamic structure function

$$
S(q ; \omega)=-\frac{\hbar}{\pi} \Im m[\chi(q ; \omega)] \theta(\omega)
$$

which satisfies, amongst others, the sum rules

$$
\begin{aligned}
& m_{0}(q)=S(q)=\int_{0}^{\infty} d \hbar \omega S(q ; \omega), \\
& m_{1}(q)=\frac{\hbar^{2} q^{2}}{2 m}=\int_{0}^{\infty} d \hbar \omega \hbar \omega S(q ; \omega),
\end{aligned}
$$


Fig. 1 (Color online)

A schematic picture of the dynamic structure function $S(q ; \omega)$ in ${ }^{3} \mathrm{He}$. The gray shaded area is the particle-hole continuum of non-interacting particles, the circles show the maximum intensity of neutron-scattering data [19] and the dots are X-ray scattering data [3]. The red line shows the results of our calculation. See also Sect. 3 for further discussions

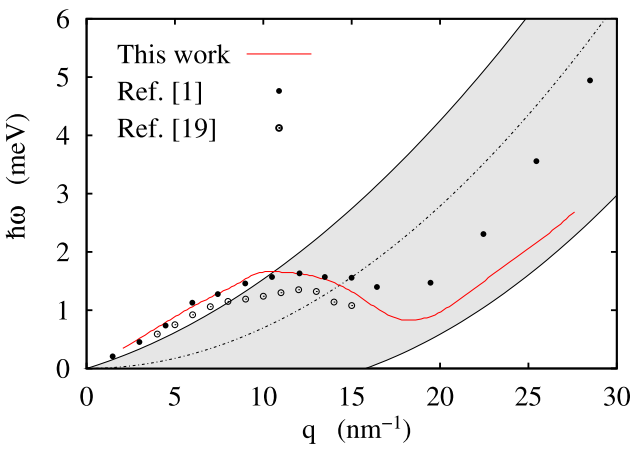

where $S(q)$ is the static structure factor. A version of the RPA with an effective interaction can be derived by restricting the excitation operator (2.7) to one-particle-one hole amplitudes, and omitting (or approximating) exchange terms.

The RPA (2.11) for $\chi(q ; \omega)$ displays the essential features of the dynamic structure function $S(q ; \omega)$ qualitatively correctly: $S(q ; \omega)$ can be characterized as being a superposition of a collective mode similar to the phonon-maxon-roton in ${ }^{4} \mathrm{He}$, plus an incoherent particle-hole band which strongly dampens this mode [18] where this is kinematically allowed. A schematic drawing is shown in Fig. 1.

The picture misses important physics: In ${ }^{3} \mathrm{He}$ the RPA, when defined through the form (2.11) and the sum rules (2.13)-(2.14), predicts a zero-sound mode that is significantly too high. This is consistent with the same deficiency of the Feynman spectrum $\varepsilon(q)=\hbar^{2} q^{2} / 2 m S(q)$ in ${ }^{4} \mathrm{He}$. Drawing on the analogy to ${ }^{4} \mathrm{He}$ [18], this deficiency can to a large extent be cured by introducing pair fluctuations $\delta u_{p p^{\prime} h h^{\prime}}^{(2)}(t)$ in the excitation operator.

In an approach that maintains the locality of the effective interactions entering the TDHF equations, we have in [13] derived practical working formulas for the effective interactions in terms of a three-body vertex and a pair propagator, these are immediately recognized as generalizations of the bosonic version:

$$
\begin{gathered}
V_{p h, p^{\prime} h^{\prime}}^{(A)}(\omega)=\frac{\delta_{\mathbf{p}+\mathbf{h}-\mathbf{p}^{\prime}-\mathbf{h}^{\prime}}}{N} \tilde{V}_{\mathrm{A}}(q ; \omega) \\
V_{p p^{\prime} h h^{\prime}, 0}^{(B)}(\omega)=\frac{\delta_{\mathbf{p}+\mathbf{p}^{\prime}-\mathbf{h}-\mathbf{h}^{\prime}}}{N} \tilde{V}_{\mathrm{B}}(q ; \omega) .
\end{gathered}
$$

The components of the (energy-dependent) interactions $V_{\mathrm{A}, \mathrm{B}}(q ; \omega)$ are

$$
\begin{aligned}
& \tilde{V}_{\mathrm{A}}(q ; \omega)=\tilde{V}_{\mathrm{p}-\mathrm{h}}(q)+\left[\sigma_{q}^{+}\right]^{2} \tilde{W}_{\mathrm{A}}(q ; \omega)+\left[\sigma_{q}^{-}\right]^{2} \tilde{W}_{\mathrm{A}}^{*}(q ;-\omega) \\
& \tilde{V}_{\mathrm{B}}(q ; \omega)=\tilde{V}_{\mathrm{p}-\mathrm{h}}(q)+\sigma_{q}^{+} \sigma_{q}^{-}\left[\tilde{W}_{\mathrm{A}}(q ; \omega)+\tilde{W}_{\mathrm{A}}^{*}(q ;-\omega)\right],
\end{aligned}
$$

with $\sigma_{q}^{ \pm} \equiv\left[S_{\mathrm{F}}(q) \pm S(q)\right] / 2 S(q)$. Here, the $\tilde{V}_{\mathrm{p}-\mathrm{h}}(q)$ is the static part of the particlehole interaction that is related to the static structure function through the RPA relationship (2.11) and the two sum rules (2.13) and (2.14). The energy dependent part of the interaction, $\tilde{W}_{\mathrm{A}}(q ; \omega)$ describes the splitting and re-combination of 
phonons, it consists of a three-phonon vertex $\tilde{K}_{q, q^{\prime} q^{\prime \prime}}$ and a two-phonon propagator $\tilde{E}^{-1}\left(q^{\prime}, q^{\prime \prime} ; \omega\right)$. The energy dependent correction to the effective interaction is

$$
\tilde{W}_{\mathrm{A}}(q ; \omega)=\frac{1}{2 N} \sum_{\mathbf{q}^{\prime}, \mathbf{q}^{\prime \prime}} \delta_{\mathbf{q}+\mathbf{q}^{\prime}+\mathbf{q}^{\prime \prime}}\left|\tilde{K}_{q, q^{\prime} q^{\prime \prime}}\right|^{2} \tilde{E}^{-1}\left(q^{\prime}, q^{\prime \prime} ; \omega\right)
$$

with a three-body vertex

$$
\begin{aligned}
& \tilde{K}_{q, q^{\prime} q^{\prime \prime}} \\
& \quad=\frac{\hbar^{2}}{2 m} \frac{S\left(q^{\prime}\right) S\left(q^{\prime \prime}\right)}{S_{\mathrm{F}}(q) S_{\mathrm{F}}\left(q^{\prime}\right) S_{\mathrm{F}}\left(q^{\prime \prime}\right)}\left[\mathbf{q} \cdot \mathbf{q}^{\prime} \tilde{X}_{\mathrm{dd}}\left(q^{\prime}\right)+\mathbf{q} \cdot \mathbf{q}^{\prime \prime} \tilde{X}_{\mathrm{dd}}\left(q^{\prime \prime}\right)-q^{2} \tilde{u}_{3}\left(q, q^{\prime}, q^{\prime \prime}\right)\right] .
\end{aligned}
$$

Here, $S_{\mathrm{F}}(q)$ is the static structure function of non-interacting fermions. $\tilde{X}_{\mathrm{dd}}(q)=$ $1 / S_{\mathrm{F}}(q)-1 / S(q)$ can be identified with the set of "non-nodal" diagrams of the Fermi-hypernetted-chain theory, and $u_{3}\left(q, q^{\prime}, q^{\prime \prime}\right)$ is the three-body ground state correlation [20].

Finally we need the pair propagator:

$$
\begin{aligned}
\tilde{E}^{-1}\left(q_{1}, q_{2} ; \omega\right) & =-\int_{-\infty}^{\infty} \frac{d \hbar \omega^{\prime}}{2 \pi \mathrm{i}} \kappa\left(q_{1} ; \omega^{\prime}\right) \kappa\left(q_{2} ; \omega-\omega^{\prime}\right) \\
\kappa(q ; \omega) & =\frac{\kappa_{0}(q ; \omega)}{1+\hbar \omega \tilde{\Gamma}_{\mathrm{dd}}(q) \kappa_{0}(q ; \omega)}
\end{aligned}
$$

with the "direct-direct" correlation function $\tilde{\Gamma}_{\mathrm{dd}}(q)=\left(S(q)-S_{\mathrm{F}}(q)\right) / S_{\mathrm{F}}^{2}(q)$, and the partial Lindhard function:

$$
\kappa_{0}(q ; \omega) \equiv \frac{1}{N} \sum_{h} \frac{\bar{n}_{\mathbf{p}} n_{\mathbf{h}}}{\hbar \omega-e_{p h}+\mathrm{i} \eta} .
$$

Evidently, we need for the execution of the theory only the static structure function $S(q)$. In this work, we have used input from the FHNC-EL calculations of [20] that were based on the Aziz-II potential [21].

\subsection{Bose Limit}

For the sake of discussions and to make the connections with previous work we briefly discuss the Bose limit. In that case, $S_{\mathrm{F}}(q)=1$, and we can identify $\tilde{\Gamma}_{\mathrm{dd}}(q) \rightarrow$ $S(q)-1$. The constituents of the pair propagator become

$$
\begin{aligned}
\kappa_{0}^{\mathrm{Bose}}(q ; \omega) & =\frac{1}{\hbar \omega-t(q)+\mathrm{i} \eta} \\
\kappa^{\mathrm{Bose}}(q ; \omega) & =\frac{1}{S(q)} \frac{1}{\hbar \omega-\varepsilon(q)+\mathrm{i} \eta} .
\end{aligned}
$$


The pair propagator is then simply

$$
\tilde{E}_{\text {Bose }}^{-1}\left(q_{1}, q_{2} ; \omega\right)=\frac{1}{S\left(q_{1}\right) S\left(q_{2}\right)} \frac{1}{\hbar \omega-\varepsilon\left(q_{1}\right)-\varepsilon\left(q_{2}\right)+\mathrm{i} \eta} .
$$

The form (2.24) shows more clearly than (2.20) the physical meaning of the pair propagator. It also shows the consequence of restricting the excitation operator to pair fluctuations: The pair propagator contains in the energy denominator the Feynman spectrum which is known to be almost a factor of two higher than the physical phonon-roton spectrum. For bosons, the problem has been cured by introducing multi-particle fluctuations $[11,12]$, these investigations show that the energy denominator gets indeed renormalized and closer to the physical spectrum. We have in the past estimated the effect of higher-order fluctuations by scaling the Feynman spectrum in the energy denominator such that the energy of the scaled spectrum agrees roughly with the experimental roton minimum, i.e. we have used

$$
\tilde{E}_{\lambda}^{-1}\left(q_{1}, q_{2} ; \omega\right)=\frac{1}{S\left(q_{1}\right) S\left(q_{2}\right)} \frac{1}{\hbar \omega-\lambda \varepsilon\left(q_{1}\right)-\lambda \varepsilon\left(q_{2}\right)+\mathrm{i} \eta}=\frac{1}{\lambda} E^{-1}\left(q_{1}, q_{2} ; \omega / \lambda\right) .
$$

We will use this procedure below to estimate the effect of multi-particle fluctuations in ${ }^{3} \mathrm{He}$.

\section{Dynamic Structure of ${ }^{3} \mathrm{He}$}

We have carried out a comprehensive array of calculations of $S(q ; \omega)$ for ${ }^{3} \mathrm{He}$ in the experimentally accessible density regime. Figure 1 shows, at saturation density, the collective mode and the particle-hole continuum corresponding to single-particle energies of non-interacting particles. Our theoretical results are compared with Xray [3] and neutron [19] scattering data. The "zero sound" mode is, when inside the particle-hole continuum, identified as the maximum value of $S(q ; \omega)$.

Figure 2 give a first glance of our results. The figures show $S(q ; \omega)$ for the densities $\rho=0.0166$ and $0.020 \AA^{-3}$, this corresponds to SVP and at a pressure of 10 bar, respectively $[22,23]$. The figures show the typical scenario of a collective mode at long wave lengths, and a particle-hole band. Note, however, that our particle-hole band does not have sharp boundaries, this effect is due to pair fluctuations which have the consequence that the interactions $\tilde{V}_{\mathrm{A}, \mathrm{B}}(q ; \omega)$ can be complex.

Recent X-ray scattering results [3] have led to a discussion on the location of the particle-hole band [4, 5]. The authors of [3] state that The obtained results show no evidence of such a decay: the zero-sound mode remains well defined in the whole explored wave number range. According to that analysis, the picture shown in Fig. 1 would not be correct, rather the particle-hole band should be significantly lower in energy. In particular the collective mode would lie outside the particle-hole band (see Fig. 3 of [3]). This is somewhat surprising because such a scenario would require a single particle spectrum that is characterized by an average effective mass $m^{*} \approx 3 m$ up to more than three times the Fermi momentum. This is in contrast to theoretical work that predicts that the effective mass should have a peak around the Fermi momentum caused by spin-fluctuations, a secondary maximum at about twice the Fermi 

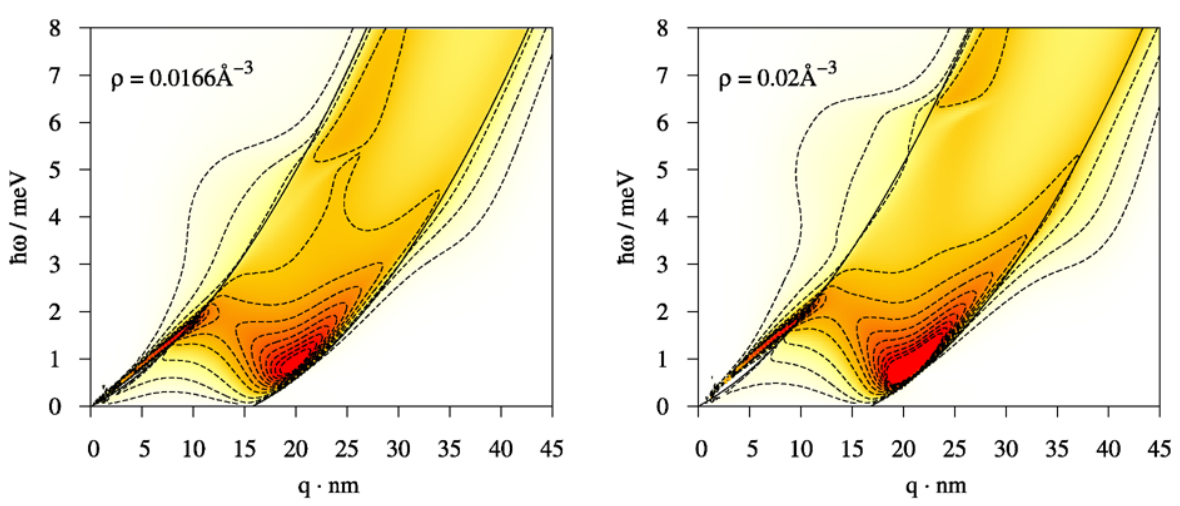

Fig. 2 (Color online) $-\Im m \chi(q ; \omega)$ at a density of $\rho=0.0166 \AA^{-3}$ (left pane) and $\rho=0.02 \AA^{-3}$ (right pane). The contour lines are at $0.02,0.05,0.1,0.2,0.3,0.4,0.5,0.6,0.7,0.8,0.9,1.0 t_{F}^{-1}$ where $t_{F}=\hbar^{2} k_{F}^{2} / 2 m$ is the Fermi energy of non-interacting ${ }^{3} \mathrm{He}$ atoms

momentum due to density fluctuations, but then falls off rapidly towards the bare mass [24-27].

Further details are shown in Fig. 3. To facilitate the comparison with experiments, we have convoluted our theoretical spectra with the experimental resolution. We have scaled our results by $1 / S(q)$ such that the integrated strength is 1 for all momentum transfers. We have also scaled the experimental spectra, the scaling factor agrees, apart from very long wave lengths, with our $1 / S(q)$.

Evidently, our results agree, after introducing the broadening, rather well with the $\mathrm{X}$-ray scattering data. It appears therefore that the X-ray data can be explained equally well by a scenario where the collective strength is inside the particle-hole band. We stress, however, that such a good agreement can not be obtained in an RPA model that assumes a form (2.11) for the density-density response function and determines the effective interaction by the sum rules (2.13) and (2.14). Typically, the strength is distributed over a wider regime, thus suggesting a broader maximum than observed.

Figure $3 \mathrm{~b}$ shows the same results for the density of $\rho=0.020 \AA^{-3}$.

\subsection{Multi-pair Coupling}

The question arises naturally whether there could be an equivalent to the "Pitaevskii plateau" [28] in ${ }^{3} \mathrm{He}$ similar to one in ${ }^{4} \mathrm{He}$. In ${ }^{4} \mathrm{He}$, this plateau is caused by the kinematic possibility that a "phonon" can decay into two "roton" excitations. The same process can, of course, also happen for fermions. However, one expects that the resulting strength is smeared out because the "roton" is per se not a sharp excitation but can be observed, at its best, by a high density of states in the area where the roton would be in a Bose system.

To determine if such an effect could be visible we recall our discussion of the Bose limit in Sect. 2.3, in particular the consequences of restricting the excitation operator (2.7) to pair fluctuations. The consequence of this version of the theory is that, in a Bose fluid, the "plateau" appears at an energy $\hbar \omega_{\mathrm{pl}}=2 \varepsilon\left(q_{\Delta}\right)$ where $q_{\Delta}$ is the wave number of the "Feynman roton", i.e. the minimum of the Feynman dispersion 

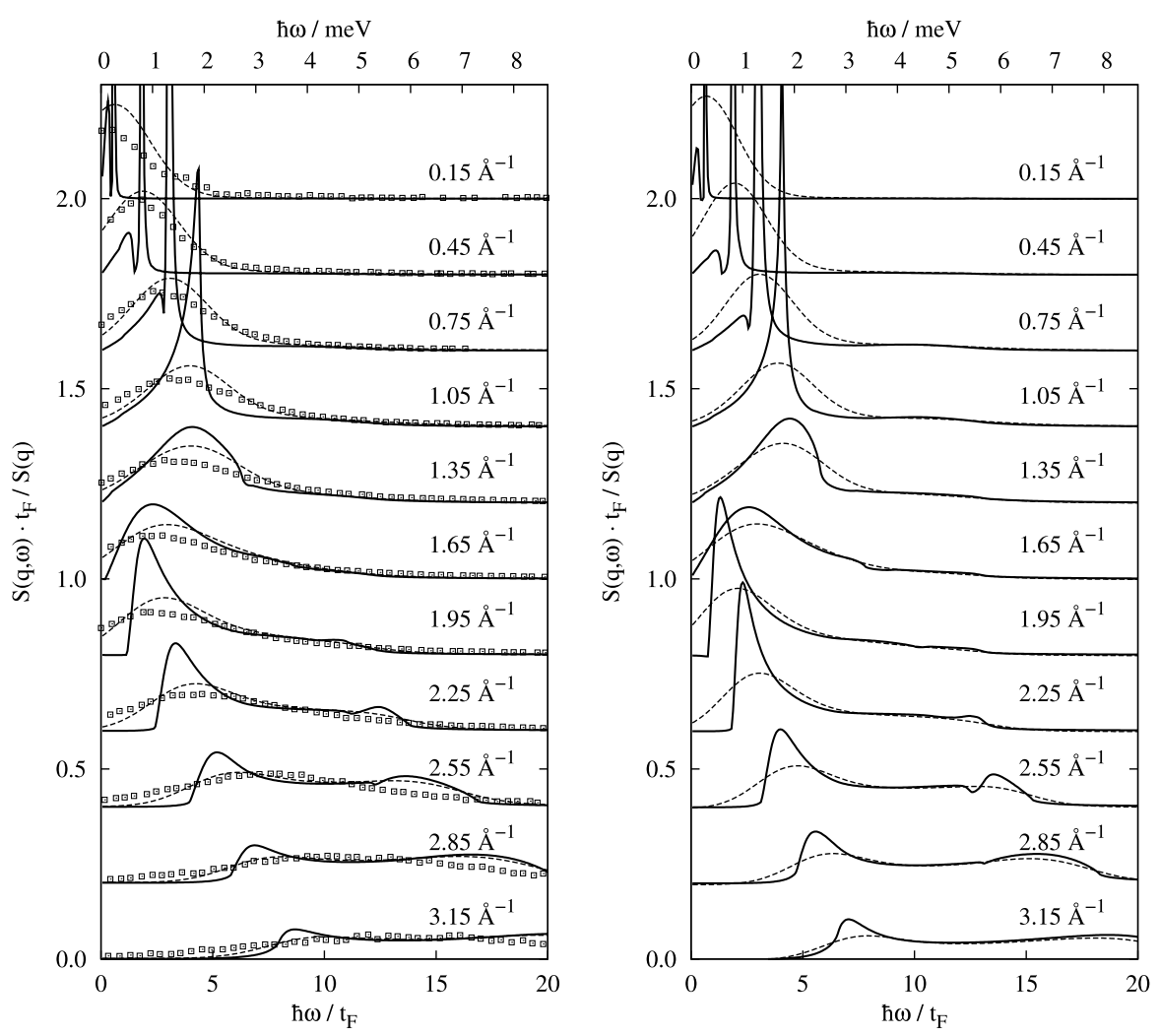

Fig. 3 The left pane shows the normalized dynamic structure function $S(q ; \omega) / S(q)$ of ${ }^{3} \mathrm{He}$ at a density of $\rho=0.0166 \AA^{-3}$, for a sequence of wave numbers. Our theoretical results (solid line) are compared with the X-ray scattering data [3] (squares) at saturated vapor pressure. Also shown are our theoretical results convoluted with the experimental resolution (dashed). The right pane shows the theory at a density of $\rho=0.02 \AA^{-3}$. All spectra are normalized to unity. The secondary maximum observed in Fig. 2 at shorter wave lengths is the due to the possible decay of excitations into two "maxon"s and will likely be broadened by multi-pair fluctuations

spectrum $\varepsilon(q)$ [10]. We should therefore also expect such a feature for fermions only at about the energy of the maximum density of states in the RPA. In both the RPA response function and in the propagator $\kappa(q ; \omega)$ this energy is of the order of $1.4 \mathrm{meV}$. The position of the "roton" is lowered by pair-fluctuations to less than $1 \mathrm{meV}, c f$. Fig. 2.

In order to estimate the consequence of including multi-particle fluctuations, we have in previous work [29] scaled the Feynman spectrum in the pair propagator such that the spectrum in the energy denominator roughly agrees with the result of the CBF calculation. Without such a scaling, one would, in the present calculation, expect a plateau-like structure at an energy of $\hbar \omega_{\mathrm{pl}} \approx 2.8 \mathrm{meV}$ and not at twice the energy of the actual "roton". We adopt here the same philosophy as in the work on bosons: We scale the two-phonon propagator as formulated in (2.25). This modification has little effect for long wave lengths, but it changes the features of $S(q ; \omega)$ for short wave 

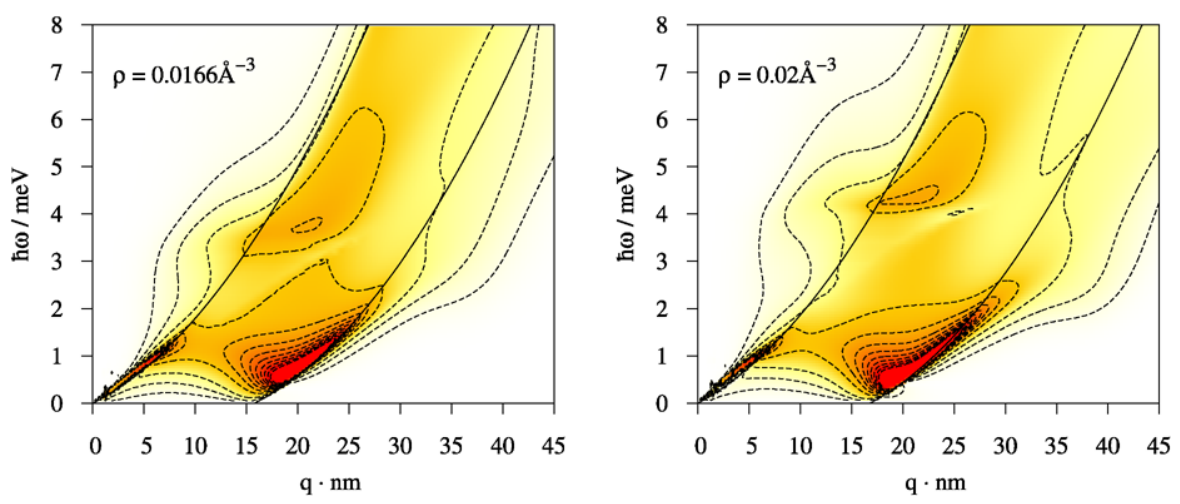

Fig. 4 (Color online) Same as Fig. 2 where the pair propagator is scaled by a factor $\lambda=0.65$ as described in $(2.25)$

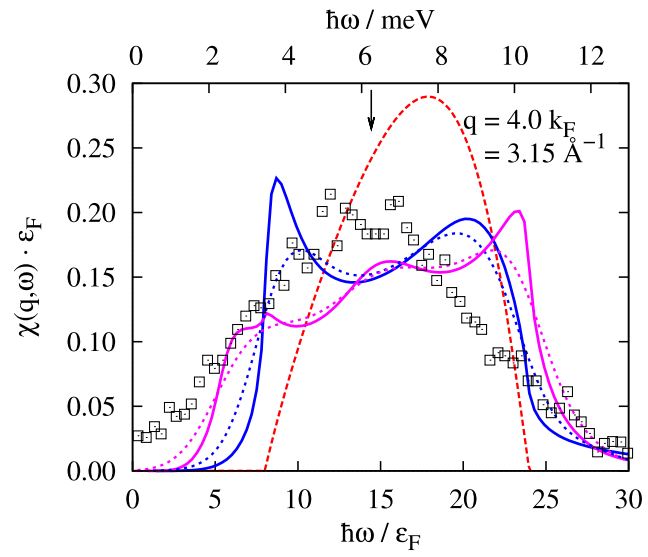

Fig. 5 (Color online) The figure shows the dynamic structure function of ${ }^{3} \mathrm{He}$ at saturated vapor pressure at $q=4 k_{F}$. The solid blue line is the result of the pair excitations theory and dashed red the RPA result. The magenta line shows the result for the pair excitations where the pair propagator has been modified by a factor $\lambda=0.65$ as described in (2.25). The corresponding dashed lines are the theoretical result folded with the experimental resolution. Inelastic X-ray diffraction data obtained by Albergamo et al. [3] are also shown (boxes)

lengths visibly as seen in Fig. 4. The most notable effect is the expected broadening of $S(q ; \omega)$ at high momentum transfers.

A closeup of the situation is given in Fig. 5 where we show $S(q ; \omega)$ at a relatively large momentum transfer $q=4 k_{F}$. Obviously the more realistic description of two pair excitations provided by the scaling procedure (2.25) has the effect of smoothing the spectrum and, in particular, adding strength to the low-energy part of the spectrum which improves the agreement between experiment and theory further. 


\section{Summary}

We have in this paper presented the application of a new theory of the dynamic structure function of Fermi systems to ${ }^{3} \mathrm{He}$. The essential results have been discussed in the text. Let us reiterate here first the basic theoretical objectives of our work: The key to a quantitatively correct description of the dynamics of ${ }^{3} \mathrm{He}$ - and, therefore, other strongly correlated quantum many-body systems - is the fact that, for excitations at atomic wave lengths, the short-ranged structure of the wave function is time dependent. The intermediate states can be described only in terms of the quantum numbers of at least two particles. With that strategy we have achieved a rather satisfactory agreement between experiment and theory.

Our results have been obtained without any modification of the particle-hole spectrum. Of course, we do not claim that the precise location of the single-particle spectrum is completely irrelevant for the energetics of the zero sound mode; but we have demonstrated that the dominant mechanism in Bose and Fermi fluids is the same, namely pair-fluctuations.

In independent work [26, 27, 30], we have used the ideas of CBF theory as well as the Aldrich-Pines pseudopotential theory to calculate the single-particle propagator in ${ }^{3} \mathrm{He}$. In both three and two dimensions, we found the above-mentioned strong variation of the effective mass with momentum. Near the Fermi surface, we found good agreement between the theoretical effective mass and the one obtained experimentally from specific heat measurements. It is, however, not clear at all if the simple concept of a single particle spectrum with a possibly momentum-dependent effective mass is appropriate away from the Fermi surface. Even simple concepts like the $\mathrm{G}(0) \mathrm{W}$ approximation leads to an energy dependent self-energy. In order to maintain the sum rules (2.13)-(2.14), any modification of the particle-hole spectrum must go along with an inclusion of exchange effects. At the level of single-pair fluctuations such a calculation is quite feasible $[31,32]$ but its implementation at the level of the theory described above still needs to be done.

In terms of the interpretation of experiments we hope that we have contributed to the clarification of X-ray scattering results. In view of the large experimental width one is inclined to consider the theoretical description as satisfactory. At longer wave lengths there are still some open questions with respect to the onset of Landau damping [19] which we hope to clarify in the future by the inclusion of the abovementioned self-energy corrections.

Acknowledgements A part of this work was done while one of us (EK) visited the Physics Department at the University at Buffalo, SUNY. Discussions with H.M. Böhm, C.E. Campbell H. Godfrin, R. Holler, and R.E. Zillich are gratefully acknowledged. This work was supported, in part, by the Austrian Science Fund FWF under project P21264.

Open Access This article is distributed under the terms of the Creative Commons Attribution Noncommercial License which permits any noncommercial use, distribution, and reproduction in any medium, provided the original author(s) and source are credited.

\section{References}

1. H. Glyde, Excitations in Liquid and Solid Helium (Oxford University Press, Oxford, 1994) 
2. H.R. Glyde, B. Fåk, N.H. van Dijk, H. Godfrin, K. Guckelsberger, R. Scherm, Phys. Rev. B 61, 1421 (2000)

3. F. Albergamo, R. Verbeni, S. Huotari, G. Vankó, G. Monaco, Phys. Rev. Lett. 99, 205301 (2007)

4. A.J.M. Schmets, W. Montfrooij, Phys. Rev. Lett. 100, 239601 (2008)

5. F. Albergamo, R. Verbeni, S. Huotari, G. Vankó, G. Monaco, Phys. Rev. Lett. 100, 239602 (2008)

6. A. Fabrocini, S. Fantoni, E. Krotscheck, Introduction to Modern Methods of Quantum Many-Body Theory and Their Applications. Advances in Quantum Many-Body Theory, vol. 7 (World Scientific, Singapore, 2002)

7. H.W. Jackson, Phys. Rev. A 8, 1529 (1973)

8. E. Feenberg, Theory of Quantum Fluids (Academic, New York, 1969)

9. C.C. Chang, C.E. Campbell, Phys. Rev. B 13, 3779 (1976)

10. C.E. Campbell, E. Krotscheck, Phys. Rev. B 80, 174501 (2009)

11. C.E. Campbell, E. Krotscheck, J. Low Temp. Phys. 158, 226 (2010)

12. C.E. Campbell, E. Krotscheck, Dynamic Many Body Theory III: Multi-particle Fluctuations in Bulk ${ }^{4} \mathrm{He}(2010$, in preparation)

13. H.M. Böhm, R. Holler, E. Krotscheck, M. Panholzer, Phys. Rev. B (2010, in press)

14. P. Kramer, M. Saraceno, Geometry of the Time-Dependent Variational Principle in Quantum Mechanics. Lecture Notes in Physics, vol. 140 (Springer, Berlin, 1981)

15. A.K. Kerman, S.E. Koonin, Ann. Phys. (NY) 100, 332 (1976)

16. D.J. Thouless, The Quantum Mechanics of Many-Body Systems, 2nd edn. (Academic Press, New York, 1972)

17. C.H. Aldrich, D. Pines, J. Low Temp. Phys. 25, 677 (1976)

18. D. Pines, Phys. Today 34, 106 (1981)

19. B. Fåk, K. Guckelsberger, R. Scherm, A. Stunault, J. Low Temp. Phys. 97, 445 (1994)

20. E. Krotscheck, J. Low Temp. Phys. 119, 103 (2000)

21. R.A. Aziz, F.R.W. McCourt, C.C.K. Wong, Mol. Phys. 61, 1487 (1987)

22. D.S. Greywall, Phys. Rev. B 33, 7520 (1986)

23. R. de Bruyn Ouboter, C.N. Yang, Physica 144B, 127 (1986)

24. V.K. Mishra, G.E. Brown, C.J. Pethick, J. Low Temp. Phys. 52, 379 (1983)

25. G.E. Brown, C.J. Pethick, A. Zaringhalam, J. Low Temp. Phys. 48, 349 (1982)

26. B.L. Friman, E. Krotscheck, Phys. Rev. Lett. 49, 1705 (1982)

27. E. Krotscheck, J. Springer, J. Low Temp. Phys. 132, 281 (2003)

28. L.P. Pitaevskii, Zh. Eksp. Teor. Fiz. 36, 1168 (1959). [Sov. Phys. JETP 9, 830 (1959)]

29. V. Apaja, E. Krotscheck, in Microscopic Approaches to Quantum Liquids in Confined Geometries, ed. by E. Krotscheck, J. Navarro (World Scientific, Singapore, 2002), pp. 205-268

30. J. Boronat, J. Casulleras, V. Grau, E. Krotscheck, J. Springer, Phys. Rev. Lett. 91, 085302 (2003)

31. N.-H. Kwong, Realistic Calculations of Excitations in Nuclear Matter. PhD thesis, California Institute of Technology, 1982

32. M. Panholzer, H.M. Böhm, R. Holler, E. Krotscheck, J. Low Temp. Phys. 158, 135 (2010) 